\title{
Nerve Growth Factor with Insular Cortical Grafts Induces Recovery of Learning and Reestablishes Graft Choline Acetyltransferase Activity
}

\author{
M.L. Escobar, N. Jiménez, J.C. López-García, R. Tapia and F. Bermúdez-Rattoni \\ Instituto de Fisiología Celular, Universidad Nacional Autónoma de México
}

\begin{abstract}
SUMMARY
Rats showing disrupted taste aversion due to insular cortex (IC)-lesions received either ICgrafts with NGF, grafts without NGF, or NGF alone. An additional group served as lesioned controls. Only those animals that received IC. grafts with NGF recovered the ability to learn the conditioned taste aversion task, at 15 days post-graft. Choline acetyltransferase (ChAT) activity in the IC-grafts with, but not without NGF, was similar to the IC activity of unoperated controls. In contrast, glutamate decarboxylase activity was similar in all the groups. These findings suggest that IC-grafts associated with NGF induce recovery of learning abilities in IC-lesioned rats, which correlates with reestablishment of ChAT activity in the grafts at 15 days postimplantation.
\end{abstract}

\section{KEY WORDS}

nerve growth factor, insular cortex, choline acetyltransferase, grafts, learning, conditioned taste aversion
It has been demonstrated that chronic perfusion of NGF in fimbria-fornix lesioned animals with severe learning impairments induces functional and anatomical recovery $/ 23 /$. Moreover, chronic intracerebral infusion of NGF improves performance in cognitively impaired aged rats $/ 24 /$. Recently Otto et al. /21/ showed that the presence of NGF-soaked gelfoam reduces the death rate of medial septal neurons following fimbria-fornix lesions.

Conditioned taste aversion (CTA) is a learning paradigm in which rats acquire aversion to a taste cue when it is followed by digestive malaise /13/. It has been shown that bilateral lesions of the insular cortex (IC) disrupt acquisition and retention of CTA /3,5,16/. Recent research in our laboratory has focused on the influence of brain grafts on the recovery of learning ability in IC-lesioned animals $12,9,10 /$. Analysis of the time course of the behavioral recovery induced by IC-grafts after IClesions, demonstrated that at the initial 15 days post-implant the subjects did not show any recuperation in the CTA paradigm, whereas a good recovery was observed at 45 days after graft. In addition, behavioral recovery was correlated with increased acetylcholinesterase activity, detected histochemically /10/ and biochemically /18/.

These findings suggest that graft maturation and/or cholinergic activity may play a role in the graft-mediated behavioral recovery following brain lesions. In the present study we evaluated the role of NGF in the recovery of CTA induced by cortical grafts, and attempted to correlate such behavioral effects with ChAT and glutamate decarboxylase (GAD) activities, as indicators of cholinergic and GABAergic neuronal systems, respectively, in the grafted tissue.

Adult male Wistar rats weighing $250-300 \mathrm{~g}$ at the start of the experiment were used. They were
Reprint address:

Federico Bermúdez-Rattoni

Instituto de Fisiología Celular

Universidad Nacional Autónoma de México

AP 70-600, 04510 México 
housed individually, under an inverted $12 \mathrm{~h}$ lightdark cycle, with food and water ad libitum except where indicated. Bilateral stereotaxic electrolytic lesions $(2 \mathrm{~mA} / 45 \mathrm{~s})$ of the IC were made in 41 animals under pentobarbital anesthesia $(50 \mathrm{mg} / \mathrm{kg}$ ) using the following coordinates with respect to bregma: $\mathrm{AP}=+1.2 \mathrm{~mm} ; \mathrm{L}= \pm 5.5 \mathrm{~mm} ; \mathrm{V}=-5.5$ $\mathrm{mm}$. Eleven animals were used as unoperated controls.

A previously described experimental procedure for CTA was used $/ 10 /$. Briefly, animals were deprived of water for $24 \mathrm{~h}$ and trained to drink water twice a day, during ten min trials for four days. On the fifth day, a $0.1 \mathrm{M} \mathrm{LiCl}$ solution, which induces digestive malaise, was given instead of water in order to induce taste aversion. After four more days of baseline consumption, the water was substituted by a $0.1 \mathrm{M} \mathrm{NaCl}$ solution to test the aversion. $\mathrm{LiCl}$ and $\mathrm{NaCl}$ are indistinguishable by rats $/ 20 \%$. The $\mathrm{NaCl}$ consumption volume was taken as the taste aversion score.

After the first behavioral CTA training and test, the experimental animals were divided randomly into five groups: Group one received IC-grafts in combination with NGF (IC-NGF, $n=13$ ); group two received IC-grafts with vehicle (IC-VEH, $n=7$ ); a third group received gelfoam soaked in the NGF solution, without graft (NGF, $n=10$ ); the fourth group was an IC-lesioned group ( $L Y, n=11)$ and the fifth was an unoperaied control group (CON, $\mathrm{n}=11$ ). Sixteen-day-old fetuses were removed from the abdominal cavity of pregnant rats under barbiturate anesthesia $(50 \mathrm{mg} / \mathrm{kg})$. The fetal brains were removed, and the temporoparietal area (above the rhinal sulcus) was dissected under a field microscope. The tissue (about $2 \mathrm{~mm}^{3}$ ) was soaked in a highly concentrated solution $(20 \mu \mathrm{g} / \mathrm{ml})$ of NGF 7S (Sigma, St. Louis, MO) in Dulbecco's Modified Eagle's Medium (GIBCO, Grand Island, NY) containing $0.25 \%$ bovine serum albumin (Sigma, St. Louis, MO), according to the procedure of Otto et al. 121\%. After soaking, the tissue was aspirated into a $100 \mu \mathrm{l}$ Hamilton microsyringe and then stereotaxically injected into the IC area, with the same stereotaxic coordinates used previously for producing the lesion. After implanting, gelfoam (about $1 \mathrm{~mm}^{3}$ ) soaked in the same NGF solution was inserted into the cavity. For group two the soaking solution and gelfoam did not contain NGF. Fifteen days after the transplantation procedure all the animals were retrained for CTA.

At the end of the behavioral procedure (postimplant) IC water homogenates from the IC region (group CON) or from dissected IC-implants (groups IC-NGF and IC-VEH) were used for measuring enzymatic activities. Animals were killed by decapitation and their brains quickly removed. The IC region was localized using the middle cerebral artery and the rhinal sulcus as references $/ 16,17 /$ and was dissected on ice and homogenized as required. The grafted tissue was carefully dissected from the host tissue and processed in the same manner. ChAT $/ 11,17 /$ and $\mathrm{GAD} / 1,17 /$ were measured by the radioisotopic techniques previously described in detail, using $\left[{ }^{3} \mathrm{H}\right]$ acetyl-coenzyme A $(1.6 \mathrm{Ci} / \mathrm{mmol})$ (NEN Dupont, Boston, MA) and $[1-14 \mathrm{C}] \mathrm{L}-$ glutamate (56 $\mathrm{mCi} / \mathrm{mmol}$ ) (Amersham, Buckinghamshire, U.K.) respectively. Protein was determined using the Folin reagent method, as described $/ 19 /$.

Two animals from each grafted group were perfused through the ascending aorta with $0.15 \mathrm{M}$ $\mathrm{NaCl}$ followed by a $4 \%$ paraformaldehyde, $0.1 \%$ glutaraldehyde solution in PBS. The brains were subsequently cut and processed for cresyl violet (Nissl staining) by standard procedures $/ 2 /$ to determine the transplants' characteristics.

A simple ANOVA was done on the test day consumption volume for all groups, pre and post graft, with post-hoc group comparisons, when appropriate, using the Student-Newmann-Keuls' test, at a 0.05 significance level. The results of the CTA experiments are shown in Fig. 1. During the pregraft test trial, there were significant differences among groups $\left(F_{4,50}=25.63, p<0.001\right)$. As expected, the control group showed strong taste aversion, whereas the IC-lesioned (IC-NGF, IC-VEH, NGF and LX) groups showed significantly disrupted taste aversion. Fifteen days after grafting, ANOVA comparisons revealed significant differences among the groups $\left(\mathrm{F}_{4,50}=11.61, \mathrm{p}<0.001\right)$. The ICNGF group showed markedly reduced $\mathrm{NaCl}$ consumption, which was similar to that of the control group, and significantly different from its pregraft score, indicating a recovery of taste

JOURNAL OF NEURAL TRANSPLANTATION \& PLASTICITY 
aversion, while the LX, NGF and IC-VEH groups did not show any improvement (Fig. 1).

The results of the determination of enzyme activities are shown in Fig. 2. ANOVA analysis of ChAT activity revealed significant differences among groups $\left(\mathrm{F}_{2,27}=9.01, \mathrm{p}<0.001\right)$. ChAT activity in IC-NGF grafts was similar to that in intact controls IC, whereas that in IC-VEH grafts was only $41 \%$ and $47 \%$, as compared with the controls and the IC-NGF tissues, respectively. In contrast, GAD activity was similar in IC homogenates in control, IC-VEH and IC-NGF groups $\left(F_{2,27}=0.56\right)$ (Fig. 2). The IC-grafts with or without NGF showed a well integrated and healthy aspect (Fig. 3).

It has been demonstrated that fetal brain grafts can produce functional recovery in a variety of behavioral tasks $/ 4,6,8 /$. We have found that in the CTA paradigm cortical brain grafts produce a significant recovery of the ability to learn after 60 days post-graft in IC-lesioned animals, and that such recovery requires at least 30 days post-graft /10/. Other studies have shown that ChAT activity in the IC is higher than in other cortical areas, and that IC-graft $\mathrm{ACh}$ release is correlated with the recovery of CTA $/ 17,18 /$, suggesting that the cholinergic system is involved in the functional recovery mediated by IC-grafts. The purpose of the present experiments was to study the effect of supplementing grafts with NGF on the behavioral recovery induced by fetal IC-implants, and to relate such effects to biochemical changes in the cholinergic or GABAergic systems.

The behavioral data obtained indicates that at 15 days post-graft the only group that promotes recovery of the ability to acquire the CTA was the group with homotopic IC-grafts in combination with NGF. In view of the above-mentioned data, this indicates that the administration of NGF in the cortical grafts significantly affects the functional recovery observed at 15 days post-graft. Experiments assessing the effects of NGF alone or in combination with grafts, in a time-dependent fashion, are currently being evaluated in our laboratory.

Our biochemical analyses showed that ChAT activity in the homotopic IC-grafts plus NGF was similar to that in the IC of intact control animals, whereas in the IC-grafts plus vehicle it was considerably reduced. Furthermore, preliminary studies using in vivo assays of ChAT and $\mathrm{ACh}$ levels have demonstrated that IC but not heterotopic grafts with NGF reestablish ChAT activity (Russell R.W., Escobar M.L., Booth R.A. and Bermúdez-Rattoni $F$., in preparation). These data, together with the above-mentioned findings, suggest a participation of cholinergic neurotransmission in the graft-mediated functional recovery. In this regard, it has been demonstrated that NGF application produces a significant regrowth and reconnectivity of cholinergic fibers using the septo-hippocampal lesion model /14/. Other authors, using different learning models and different brain regions, have found recovery after acute application of NGF or other trophic factors. Moreover, that recovery has been correlated with the survival and improvement of the functional capabilities of cholinergic cells $/ 7,12,15,22,24 /$. In this regard, Varon and coworkers /23/ using chronic NGF administration in animals with fornix lesions, have described functional recovery in a Morris spatial task, that also correlated with regrowth of cholinergic fibers.

In the present experiments, measurement of GAD activity at 15 days post-graft, in contrast to ChAT, did not show any significant differences between groups. These results suggest that GABA mediated neurotransmission does not play an important role in graft-promoted behavioral recovery. Obviously, the involvement of other neurotransmitter systems cannot be dismissed. Despite the apparent similarity in the morphological appearance of the IC-NGF and ICVEH grafts, the results indicate that the biochemical differences in ChAT activity are more relevant to the behavioral recovery observed.

The present findings suggest that NGF, when associated simultaneously with homotopic ICgrafts, produces recovery of learning abilities in IC-lesioned rats and the reestablishment of ChAT activity in the grafts at 15 days post-graft.

\section{ACKNOWLEDGEMENTS}

The present research was supported by DGAPA-UNAM IN 204689: CONACYT 0178- 


\section{GONDITIONED TASTE AVERSION}

\section{PRE-GRAFT TEST}

\section{POST-GRAFT TEST}
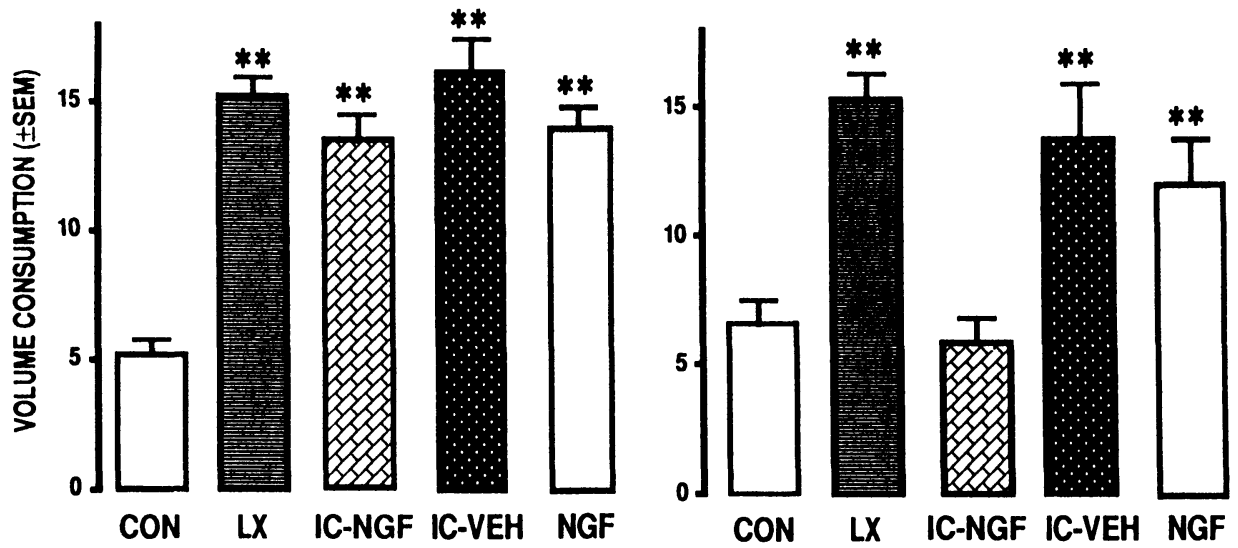

Fig. 1: Amount of $\mathrm{NaCl}$ consumed by control (CON), lesioned (LX), insular cortex + NGF graft (IC-NGF), NGF alone (NGF) and insular cortex + vehicle graft (IC-VEH) groups. Left panel shows the results from one taste test trial prior to implant. Right panel shows the results of one taste test trial 15 days after implant. Values are means \pm SEM. ${ }^{* *}$ p $<0.01$ as compared with controls (Newmann-Keuls' test).
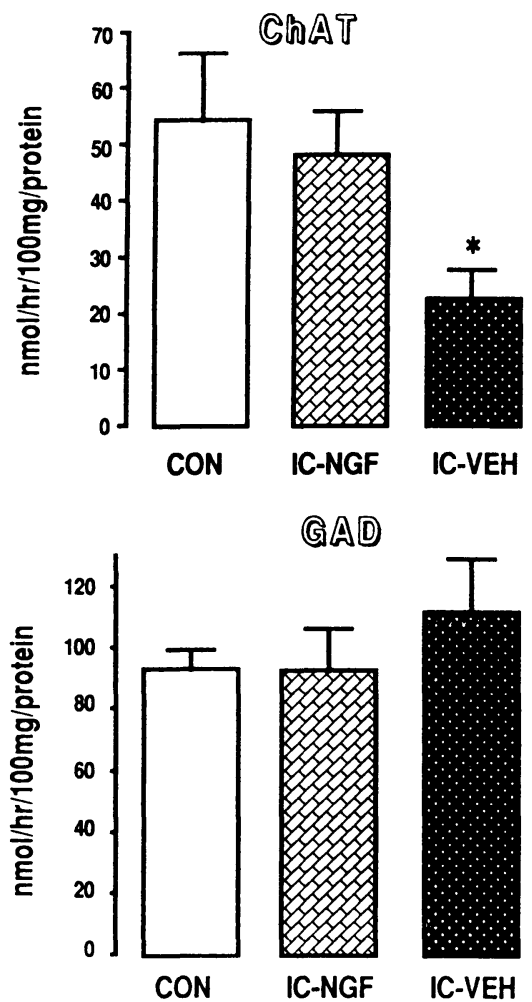

Fig. 2: ChAT (top panel) and GAD (bottom panel) activity in IC of intact control (CON), IC-NGF and IC-VEH grafts. ${ }^{*} \mathrm{p}<0.05$ as compared with both control and IC-NGF groups (Newmann-Keuls' test). 


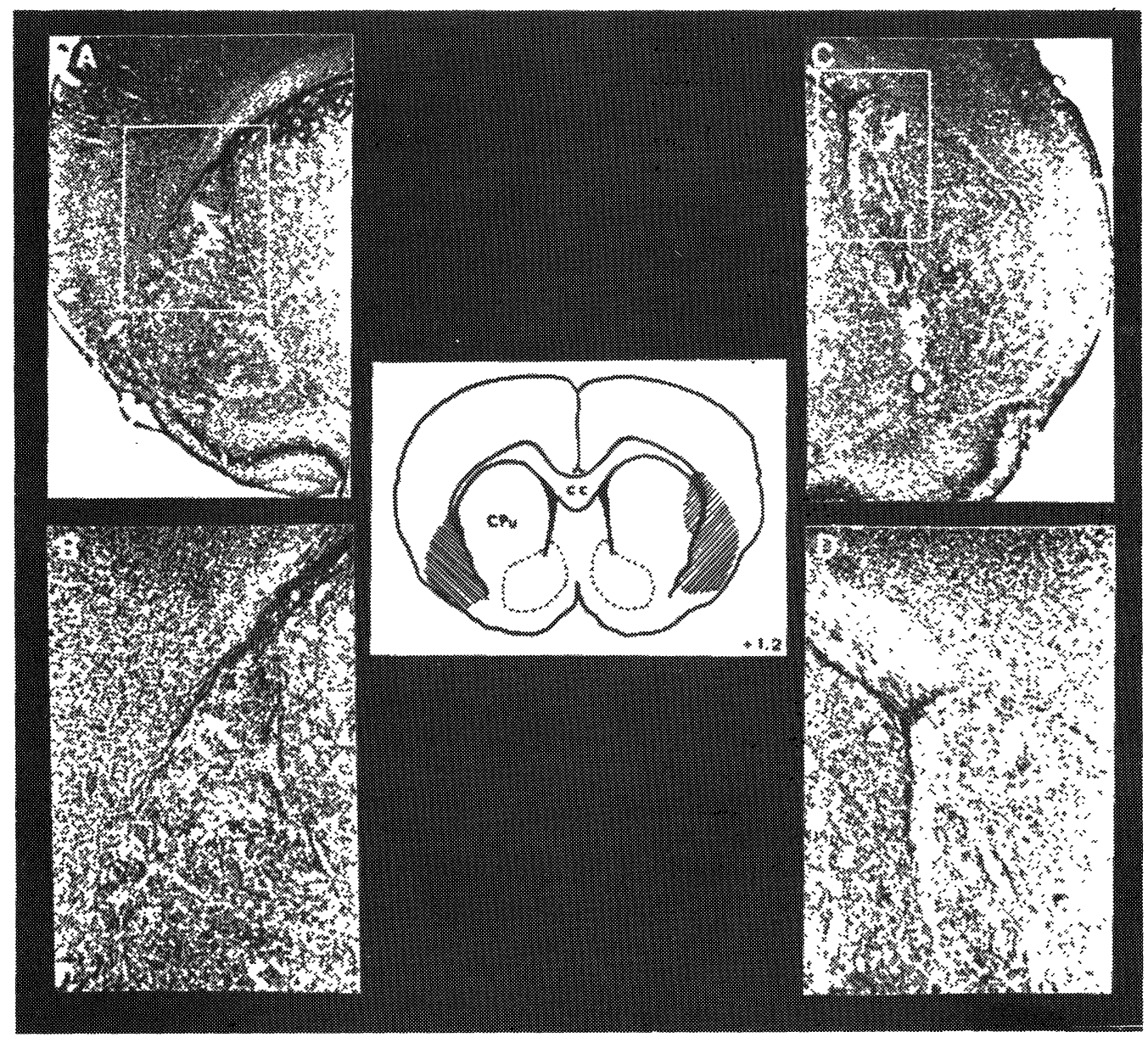

Fig. 3: Nissl staining of coronal sections from homotopic insular cortex grafts with (A and $B$ ) or without NGF (C and D). The position of the grafts in the host brain is schematized (center). B and D (40x) magnifications of the boxed regions in A and $C(20 x)$ respectively.

N9107 and PADEP-UNAM-DCCH9176. We thank Dr. M Hiriart for her contribution and Mr. Oreste Carbajal for his technical assistance.

\section{REFERENCES}

1. Albers RW, Brady RD. The distribution of glutamic acid decarboxylase in the nervous system of the Rhesus monkey. J Biol Chem 1959; 234: 926-928.

2. Bermúdez-Rattoni F, Fernández J, Sánchez MA, Aguilar-Roblero R, Drucker-Colín R. Fetal brain transplants induce recuperation of taste aversion learning. Brain Res 1987; 416: 147-152.

3. Bermúdez-Rattoni F, McGaugh JL. Insular cortex and amygdala lesions differentially affect acquisition on inhibitory avoidance and conditioned taste aversion. Brain Res 1991; 549: 165-170.

4. Bermúdez-Rattoni F, Escobar ML, Piña AL, Tapia R, López-García JC, Hiriart M. Effects of NGF on the recovery of conditioned taste aversion in the insular cortex lesioned rats. In: Doty RL, ed, Chemical Signals in Vertebrate VI, Plenum Press 1992; 297-303.

5. Braun JJ, Lasiter PS, Kiefer SW. The gustatory neocortex of the rat. Physiol Psychol 1982; 10: 13-45.

6. Collier TJ, Gash DM, Sladek JR. Transplantation of NE neurons into aged improves performance of a learned task. Brain Res 1988; 448: 77-87.

7. Davies SW, Beardsall K. Nerve growth factor selectively prevents excitotoxin induced degeneration of striatal cholinergic neurons. Neurosci Lett 1992; 140: 161-164.

8. Dunnett SB, Ryan CN, Levin PD, Reynolds M, Björklund A. Functional consequences of embryonic neocortex transplanted to rats with prefrontal cortex lesions. Behav Neurosci 1987; 101: 489-503.

9. Escobar M, Fernández-Ruíz J, Guevara-Aguilar R, Bermúdez-Rattoni F. Fetal brain grafts induce recovery of learning deficits and connectivity in rats with gustatory neocortex lesion. Brain Res 1989; 478: 368374.

10. Fernández-Ruíz J, Escobar ML, Piña AL, Díaz-Cintra $\mathrm{S}$, Cintra-McGlone FL, Bermúdez-Rattoni F. Time 
dependent recovery of taste aversion learning by fetal brain transplants in gustatory neocortex-lesioned rats. Behav Neural Biol 1991; 55: 179-193.

11. Fonum F. A rapid radiochemical method for the determination of choline acetyltransferase. J Neurochem 1975; 24: 407-409.

12. Gage FH. NGF-dependent sprouting and regeneration in the hippocampus. Prog Brain Res 1990; 83: 357-370.

13. García J, Lasiter PS, Bermúdez-Rattoni F, Deems DA. General theory of aversion learning. Ann NY Acad Sci 1985; 443: 8-20.

14. Hagg T, Vahlsing HL, Manthorpe M, Varon S. Nerve growth factor infusion into denervated adult rat hippocampal formation promotes its cholinergic reinnervation. J Neurosci 1990; 10: 3087-3092.

15. Hefti F. Nerve growth factor promotes survival of septal cholinergic neurons after transection. J Neurosci 1986; 6: 2155-2162.

16. Lasiter PS, Glanzman DI. Cortical substrates of taste aversion learning: involvement of the dorsolateral amygdaloid nuclei and temporal neocortex in taste aversion learning. Behav Neurosci 1983; 99: 257-276.

17. López-García JC, Bermúdez-Rattoni F, Tapia R. Release of acetylcholine, $\gamma$-aminobutyrate, dopamine, acid glutamate, and activity of some related enzymes in rat gustatory neocortex. Brain Res 1990; 523: 100-104.

18. López-García JC, Fernández-Ruíz J, Bermúdez-Rattoni
F, Tapia R. Correlation between acetylcholine release and recovery of conditioned taste aversion induced by fetal neocortex grafts. Brain Res 1990; 523: 105-110.

19. Lowry O, Rosebrough N, Farr A, Randall RJ. Protein measurement with the Folin phenol reagent. J Biol Chem 1951; 193: 265-275.

20. Nachman M. Learned aversion to the taste of lithium chloride and generation to other salts. J Comp Physiol 1963: 56: 343-349.

21. Otto D, Frotscher M, Unsicker K. Basic fibroblast growth factor and nerve growth factor administered in gelfoam rescue medial septal neurons after fimbria fornix transection. J Neurosci Res 1989; 22: 83-91.

22. Rosenberg MB, Friedman $T$, Robertson RC, Tuszynski M, Wolff JA, Breakefield XO, Gage FH. Graft in genetically modified cells to the damaged brain; Restorative effects of NGF expression. Science 1988; 242: $1575-1578$.

23. Varon S, Hagg T, Vahlsing L, Manthorpe M. Nerve growth factor in vivo actions on cholinergic neurons in the adult rat CNS. In: Todd LE, Packer L, Jaz J, eds, Cell Function and Disease. Plenum Press, 1989; 235248.

24. Will B, Hefti F. Behavioral and neurochemical effects of chronic intraventricular injections of nerve growth factor in adult rats with fimbria lesions. Behav Brain Res 1985; 17: 17-24. 

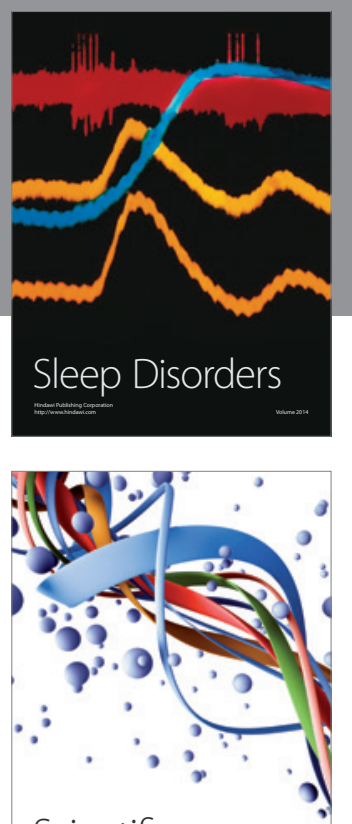

Scientifica
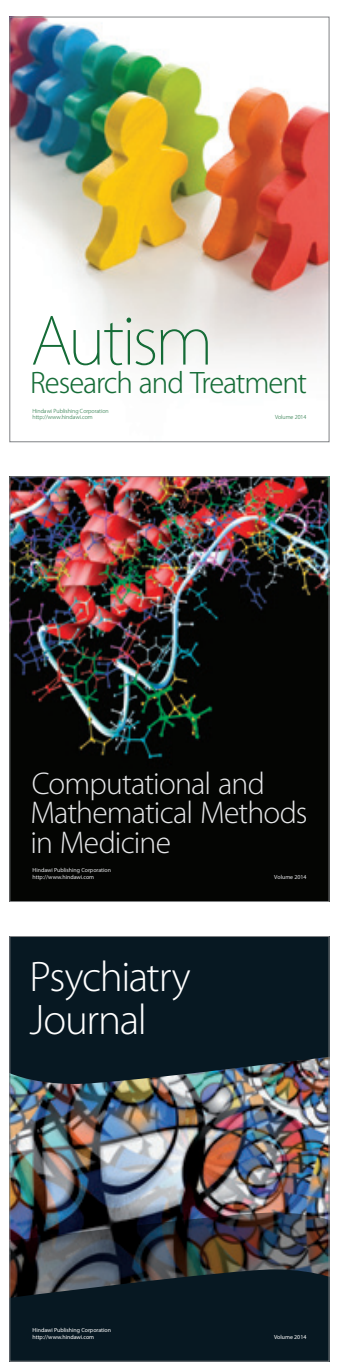
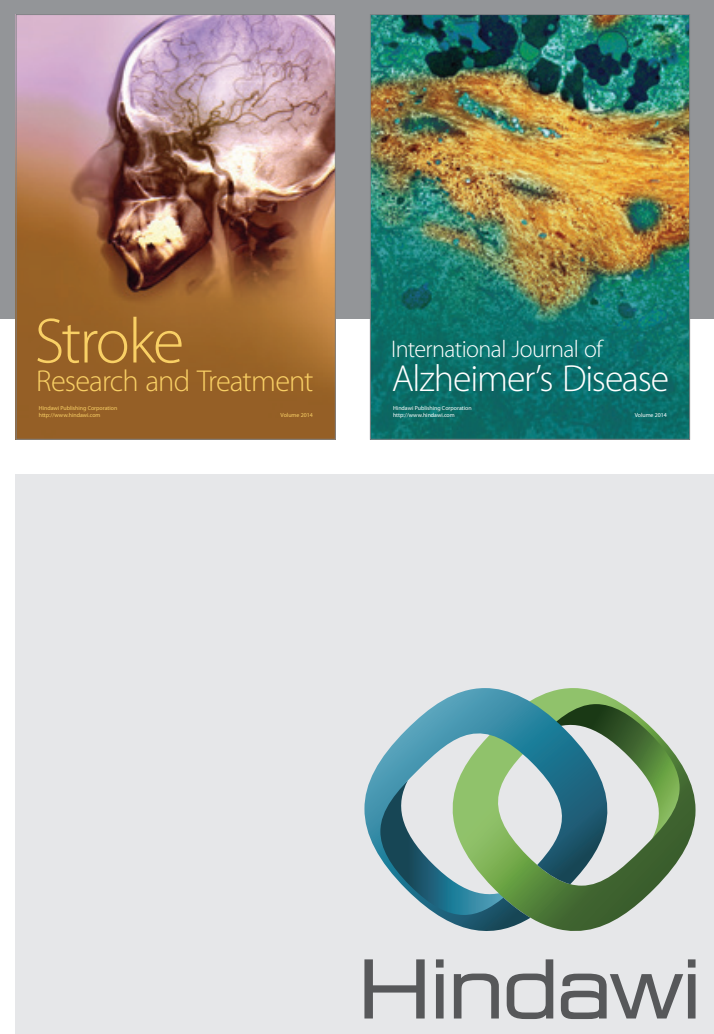

Submit your manuscripts at

http://www.hindawi.com
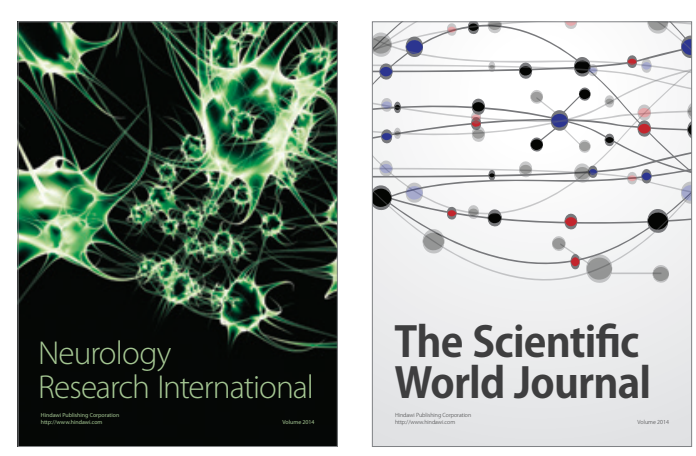

The Scientific World Journal

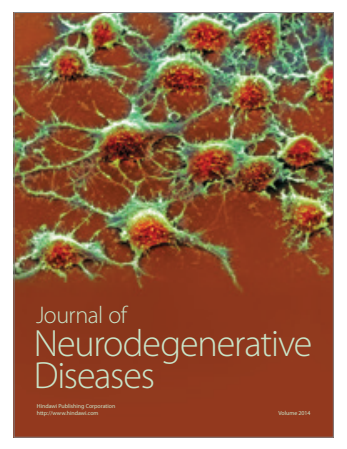

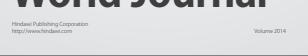

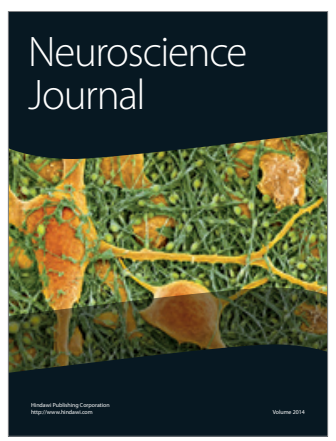

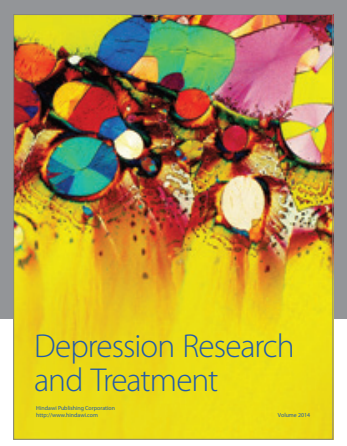
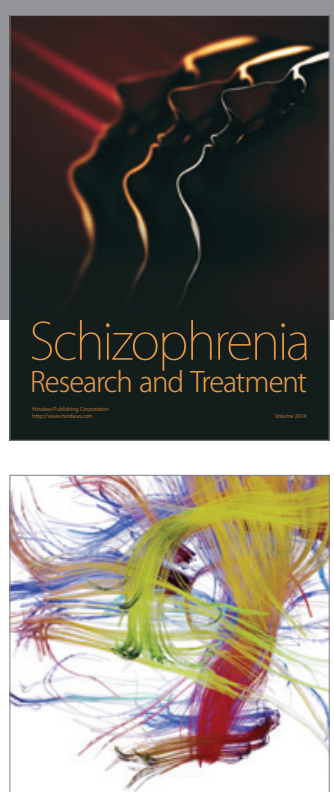

Brain Science

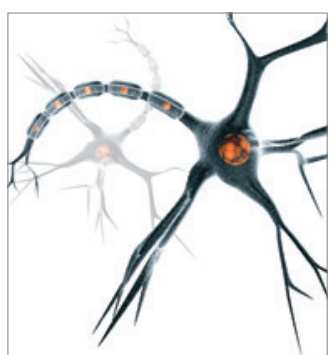

Neural Plasticity
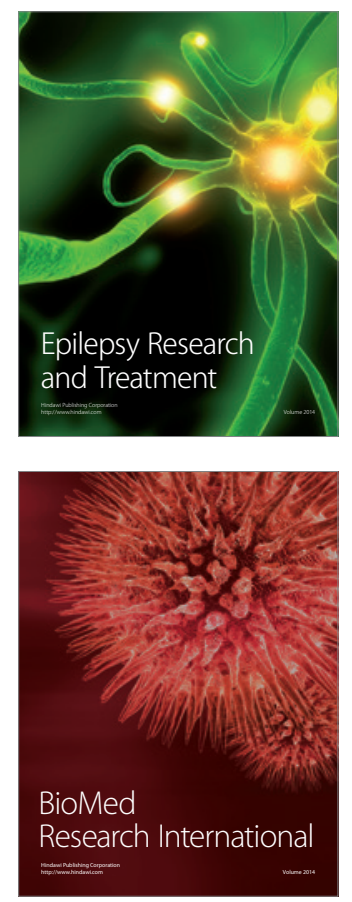

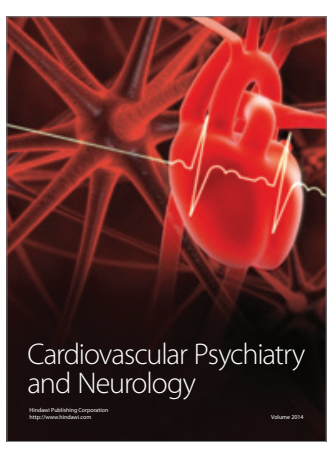

Parkinson's

Disease
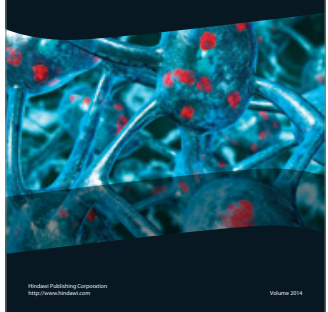